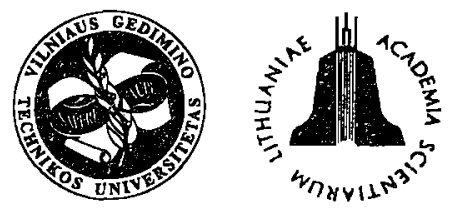

ISSN 1648-4142 TRANSPORT

http:/www.vtu.lt/english/editions

\title{
MODELLING OF FREIGHT FLOWS THAT CONSIST OF MULTIPLE PRODUCTS TRANSPORTED BY DIFFERENT MODES OF TRANSPORT IN A MULTIMODAL NETWORK
}
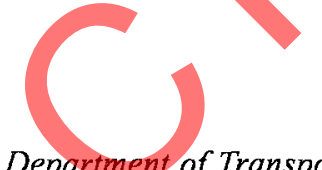 \\ Aidas Vasilis Vasiliauskas \\ Department of Transport Management, Vilnius Gediminas Technical University, \\ Plytines g.27,LT-2040 Vilnius, Lithuania,Tel. (370) 5 2699694.E-mail: aidasv@ti.vtu.lt \\ Received 200205 07; accepted 20020910
}

Abstract. Freight flows which consist of multiple products transported by different modes of transport in a multimodal network get more and more attention in recent years. In this article a model for modelling flows of multiple products in the multimodal network is presented. The multimodal aspect of considered transport system is accounted by the chosen means of network representation. The multiproduct aspect is estimated by the solution procedure which is a Gauss-

Seidel-Linear Approximation Algorithm. The article is closely connected with the following topics:

- the representation of the network chosen for the reflection of transportation and transfer operations;

- the formulation of a multimode multiproduct model;

- the analysis of average and marginal cost functions;

- the development of the solution algorithm;

- the development of the algorithm used to compute the shortest path.

Keywords: freight flows, multiple products, multimodal network, different modes of transport

\section{Introduction}

Flows which consist of multiple products that move in a multimodal network get more and more attention in recent years. The class of models that has to do with the prediction of interregional freight flows was well studied in the past and is so-called the spatial price equilibrium models [1]. Such models were widely used for the analysis of freight flows between different regions. This class of models determines simultaneously the flows between producing and consuming places as well as the selling and buying prices. But the transportation network is usually modelled in a simple way (elementary bipartite connections) [1, 2]. For example in the Harvard Model which probably is the first published type of a model that interests us physical network is presented like elementary direct links. Also the congestion effect of the network in this model is not considered. Later, the Multi-State Transportation Corridor Model went a step further in representing an explicit multimodal network, but again without any consideration of network congestion. Also there was an Transportation Network Model which does not consider network congestion effect either [1].

The first model that considers the network congestion effect as well as the shipper-carrier interaction and was adopted in the field of transport, is the Freight Network Equilibrium Model [1-3]. It combines the variable demand modelling approach from spatial equilibrium models with the detailed description of the behavior of shippers and carriers by mathematical formulations.
So the class of models that we consider is Network Models. They enable the prediction of flows that consist of multiple products in a multimodal network, when physical network is modelled at a level of detail appropriate for a country or a large region and reflects the real infrastructure with relatively little abstraction. Also in this class of models the shippers and the carriers are not considered as distinct actors in the decisions made in shipping freight. Very often the strategic analysis of freight transport is determined by the data sources (national freight flow statistics) which permit to identify the mode used, but do not provide any information about separate shippers. However, even with the data which is available and with the forecast made the behavior of the shippers can be reflected [1-3].

In the model an assumption that products are transported at minimum total generalized cost is made [4].

Also there is an assumption that a certain type of products can be transported only by the appropriate mode of transport due to service availability or some kind of normative restrictions. This means that in most common situation there is no competition between transport modes. In other situation, when transport modes compete between themselves for the shipments of products, it is possible to include the components which reflect the shippers objectives in to the generalized cost functions. This means that the generalized cost function gives sufficient modelling flexibility and adequately reflects a wide variety of situations and circumstances $[4,5]$. 
The multimode multiproduct model is formulated in the most general way permitting in principle non-convex and asymmetric cost functions. Nevertheless, certain as sumptions (regarding the structure of cost functions) are made in order to simplify the problem.

\section{The Network Representation}

The physical network infrastructure represented by the chosen means reflects the transportation of different products by several different transport modes.

A product is any good or collection of goods of similar nature which creates a freight flow that moves over a particular link $[4,6,7]$.

A transport mode is a group of transport means that has its own characteristics such as vehicle type, capacity, and a specific cost function. Depending on the detailed level of study our model mode can represent a particular carrier, or a part of his service on a specific network, or an aggregated service of several carriers in specific transport infrastructure networks $[4,6-8]$.

Modelled network is a network that consists of the nodes, links and modes of transportation that show all possible movements on the available infrastructure. In our model a link is defined as a triplet $(i, j, m)$, where is the origin node, $\mathrm{i}$ belongs to $\mathrm{N}(\mathrm{N}$ is the set of the nodes of the network), $\mathrm{j}$ is the destination node, $\mathrm{j}$ belongs to $\mathrm{N}, \mathrm{m}$ is the mode of transport that has permission to operate between nodes $i$ and $j$ and belongs to $M$ ( $M$ is the set of transport modes available on the network). Example of such network is shown in Fig 1. Here are 3 transport modes: road transport $R$, diesel train $D$, electrified train $E$, and three nodes of the network A, B, C. All 3 transport modes operate between points $A$ and $B$, between $A$ and $C$ road and diesel train services can be found, and between $B$ and $C$ only road transport service can be found.

The most compact way to represent the physical network would be to connect all the points by direct links and to allocate transport modes as link attributes exactly as shown in Fig 2 a.

But this network representation has some major disadvantages.

$\mathbf{A}$
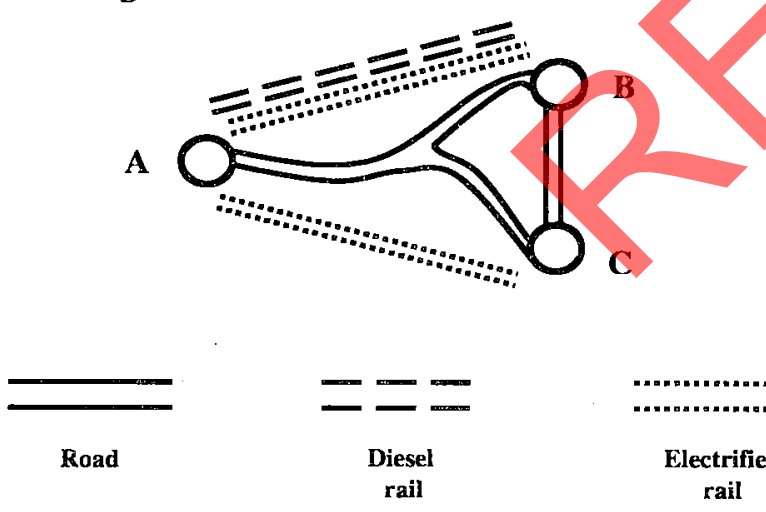

Fig 1. Physical network
If a single flow is associated with a specific link, it must be the total flow on that link. But then the flows specific to each transport mode are not determined explicitly.

If flows are separate one for each mode and are associated with each link, then these flows can vary in number from link to link depending on the number of transport modes operating on a given link. In addition, the physical difference of infrastructure modeled is not explicit in such representation. Thus the specification of cost functions for each transport mode possesses the same problem as that mentioned above for the flows [3, 6, 9-11].

In order to overcome these drawbacks it is a must to choose a network representation which permits the identification of the flows and the cost functions by the separate transport mode. This would mean the establishment of the detailed network "copy" for each separate transport mode used in the movements of products. However, this type of network representation becomes hard enough to put into practice, if large networks with many transport modes have to be considered [12].

That's why the best way for multimodal network representation is to permit the parallel links, one for each permitted transport mode that can move between each pair of nodes. In this way the network model becomes similar to real network because, for example, even though rail and road transport networks are located between the same two points, they are physically different. Besides that, if on a physical link, such as rail road, there are two different types of services (diesel and electrified trains), separate links must be assigned for each service, since they have different cost functions [4]. All this is shown in Fig 2 b.

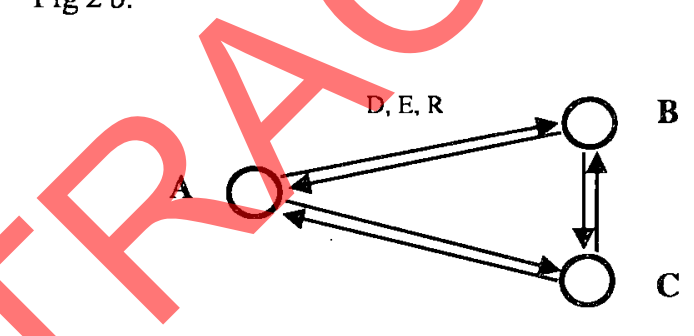

a)

A

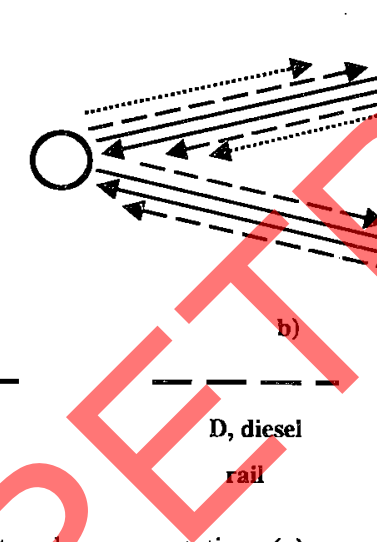

Fig 2. Network representation: (a) compact and (b) parallel 
When the network representation is chosen, in order to model intermodal shipments further it is necessary to associate the appropriate cost functions for transfer operations from one mode to other at the certain network nodes. All this can be done expanding a node: add as many extra nodes (and links between them) as there are possible transfers between transport modes that serve that node. Example is shown in Fig 3.

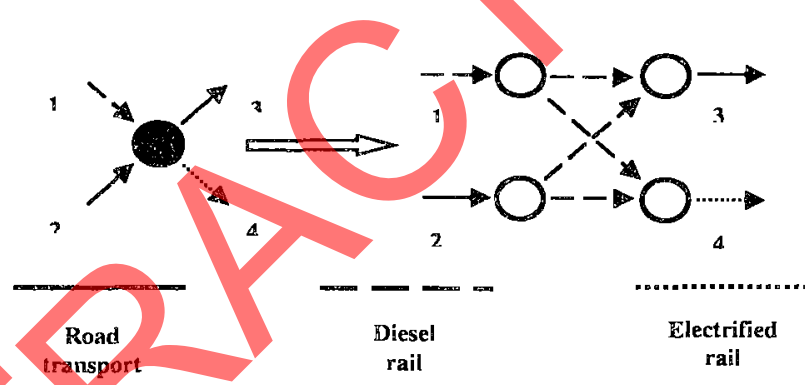

Fig 3. Explosion of a node

It must be noted, that:

Such expanding of nodes increases the number of links in the network;

- Only new nodes do not reflect the real network and new links in the node do that;

- New (transfer) links unlike the other network links reflect the transfers from one transport mode to another, so they can not be attributed to one or another transport mode;

- The representation chosen to reflect the transport operations does not require the explicit modification of a network.

It is of great importance to point out several advantages of the chosen network representation. In such network the total path consists of a sequence of links of the first transport mode, a possible transfer links to another mode, a sequence of links of the second transport mode, and so on. This means that transport mode change is possible only at transfer nodes. This representation also permits to restrict the movements of a certain type of products only by the appropriate transport modes [8].

\section{Formulation of Multimodal Multiproduct Model}

The most efficient way to use the transport infrastructure is to transport the freight at the least total cost. Unfortunately, a variety of circumstances prevents the achievement of that goal $[3,9,10,13,14]$. So with the model that we are trying to formulate there is a try to minimize total costs.

The network that we consider consists of a set of nodes $\mathrm{N}$, a set of links $\mathrm{A}, \mathrm{A} \subset \mathrm{N} \times \mathrm{N} \times \mathrm{M}$, a set of modes $M$, and set of transfer links $T, T \subset A \times A$. We denote their cardinality $\eta_{N} \eta_{A} \eta_{M}$ ir $\eta_{T}$ respectively. With each link $\alpha$, $\alpha \in \mathrm{A}$ we associate a cost function $\mathrm{s}_{\alpha}($.$) which depends$ on the volume of goods on that link or possibly, on the volume of goods on the other links of the network. Similarly, a cost function $\mathrm{s}_{\mathrm{t}}($.$) is associated with each transfer$ link $t, t \in T$.

Products that are the part of the freight flow transported over a multimodal network are denoted by $\rho, \rho \in P$, where $P$ is a set of all products and its cardinality is $\eta_{p}$.

Every product is transported from origin $0,0 \in O \subseteq$ $\mathrm{N}$ to destination $\mathrm{d}, \mathrm{d} \in \mathrm{D} \subseteq \mathrm{N}$ point. The demand of each product for all origin/destination points pairs $\mathrm{O} / \mathrm{D}$ is specified by the set of $\mathrm{O} / \mathrm{D}$ matrices. The transport mode choice for the transportation of each product is indicated defining a sub set of transport modes permitted to transport the corresponding demand for each of these $\mathrm{O} / \mathrm{D}$ matrices.

Let $g^{m}(p)$ be the demand matrix associated with the product $p, p \in P$, and $m(p)$ is a sub set of transport modes that belongs to $M(p)$ - set of all sub sets of transport modes that are used to transport product $p$.

The flow of product $\mathrm{p}$ in a multimodal network is denoted by $v^{\mathrm{p}}$ and consists of the flows of this product on links and transfer links.

$$
v^{p}=\left(\begin{array}{ll}
\left(v_{a}^{p}\right), & a \in A \\
\left(v_{t}^{p}\right), & t \in T
\end{array}\right) .
$$

The flow of all products in a multimodal network is denoted by $v$ and is a vector of dimension $\eta_{\rho}\left(\eta_{A}+\eta_{T}\right)$.

The average cost function $\mathrm{s}_{\mathrm{a}}^{\mathrm{p}}(\mathrm{v})$ on links and $\mathrm{s}_{\mathrm{t}}{ }_{\mathrm{t}}(\mathrm{v})$ on the transfer links correspond to a given flow vector. The average cost function for the product $p$ is denoted similarly to the denotation used for the flows $-\mathrm{s}^{\mathrm{p}}$ :

$$
\left.s^{p}=\left(\begin{array}{ll}
\left(s_{a}^{p}\right. \\
s_{t}^{p}
\end{array}\right), \quad, \quad, \quad t \in T\right) .
$$

And so $s$ is a vector of average cost function of dimension $\eta_{\rho}\left(\eta_{A} \times \eta_{T}\right)$.

The total cost of the flow of all products p on the link $\alpha$ is a product $s_{\mathrm{a}}^{\mathrm{p}}(v) v_{\mathrm{a}}^{\mathrm{p}}$, and the total cost of flow of product on the transfer link $t$ is product $s_{t}^{p}(v) v_{t}^{p}$. So the total cost of the flows of all products in the multimodal network is the function $\mathrm{F}$, which expression is:

$$
F=\sum_{p \in P}\left(\sum_{a \in A} s_{a}^{p}(v) v_{a}^{p}+\sum_{t \in T} s_{t}^{p}(v) v_{t}^{p}\right) \text {. (3) }
$$

$F$ is that function that we are seeking to minimize. But it is well known that in order to do this we must have some constraints. In our case this can be the conservation of the flow and non-negativity constraints. In order to write these two constraints we shall use the following denotation. Let $K_{o d}^{m(p)}$ denote the set of paths that goes from the origin point $o$ to the destination point $d$ using only transport mode $m(p)$. Then the equation of the conservation of flow is expressed:

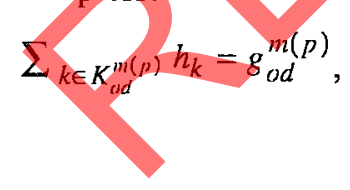


$h_{k}$ is the flow on the path $k$.

Non-negativity constraints then are:

$$
h_{k} \geq 0 \text {. }
$$

Let $\Omega$ be the set of flows that satisfies (4) and (5). Since the constraint (4) is stated in the space of path allows, for notational convenience the specification of $\Omega$ requires the relation between link flows and path flows, which is:

$$
\begin{aligned}
& v_{a}^{p}=\sum_{k \in K^{p}} \delta_{a k} h_{k}, a \in A, p \in P \\
& K^{p}=\bigcup_{m(p) \in M(p)} \cup_{O \in O} \bigcup_{d \in D} K_{o d}^{m(p)} \text { is a set of }
\end{aligned}
$$

all paths through which product $\mathrm{p}$ can be transported.

$$
\delta_{a k}=\left\{\begin{array}{ll}
1 & \text { jei } a \in k \\
0 & \text { other way }
\end{array}\right. \text { is the indicator function which }
$$

identifies the links of particular path.

Similarly, the flows on a transfer links are:

$$
v_{t}^{p}=\sum_{k \in K^{p}} \delta_{t k} h_{k}, t \in T, p \in P .
$$

$$
\text { Here } \delta_{a k}=\left\{\begin{array}{lll}
1 & \text { jei } t \in k \\
0 & \text { other way. }
\end{array}\right.
$$

So in conclusion it can be said that we developed an optimal multiproduct multimodal assignment model which consists of a function (3) that we are seeking to minimize regarding constraints (4) and (5).

It should be noted that the model is sufficiently general. But it is clearly understood that the purpose of strategic planning is not the detail model that identifies shippers and carriers explicitly. The goal was to develop a model that is adequate for the comparison of scenarios a model which helps to study the process of distribution of multiple products with the help of different modes of transport in a multimodal network.

\section{Analysis of Average and Marginal Cost Functions}

In the model formulated in the previous section the average cost functions $s_{a}(v)$ and $s_{t}(v)$ in principle may depend on any or all the components of the vector $v$. Yet, the actual cost functions on the network links and transfer links are in principle different (exception is transportation services that share the same facilities, e.g. rail services that operate in both directions on a single track) $[5$, $9,10,13,14]$

Next we state the simplified form of the average cost function. The most general expression form of the marginal costs for transporting product $\bar{p}$ on link $\bar{a}$ is:

$$
c \frac{\bar{p}}{a}=s_{\bar{a}}^{\bar{p}}+\sum_{p \in P}\left(\sum_{a \in A} \frac{\delta s_{a}^{p}}{\delta v_{\bar{a}}^{\bar{p}}} v_{a}^{p}+\sum_{t \in T} \frac{\delta s_{t}^{p}}{\delta v_{\bar{a}}^{\bar{p}}} v_{t}^{p}\right)
$$

The expression of the marginal costs for transporting products $\bar{p}$ on the transfer link $t$ is:

$$
c_{\bar{t}}^{\bar{p}}=s_{\bar{t}}^{\bar{p}}+\sum_{p \in P}\left(\sum_{a \in A} \frac{\delta s_{a}^{p}}{\delta v \frac{\bar{p}}{\bar{p}}} v_{a}^{p}+\sum_{t \in T} \frac{\delta s_{t}^{p}}{\delta v_{\bar{t}}^{\bar{p}}} v_{t}^{p}\right) .
$$

Next we make the following assumptions regarding the average cost functions:

1. The link cost functions do not depend on the flows on the transfer links.

2. The transfer link cost functions do not depend on the flows on the network links.

3. The transfer links cost functions do not depend on the flows on the other transfer links.

These assumptions are quite natural and result in the simplification of equation (8) to expression:

$$
c c_{\bar{a}}^{\bar{p}}=s_{\frac{\bar{p}}{a}}+\sum_{p \in P} \sum_{a \in A} \frac{\delta s_{a}^{p}}{\delta v_{\frac{\bar{p}}{a}}^{\bar{p}}} \cdot v_{a}^{p} .
$$

The same simplification can be done with the equaion (9):

$$
c_{\bar{t}}^{\bar{p}}=s_{\bar{t}}^{\bar{p}}+\sum_{p \in P} \frac{\delta s_{\bar{t}}^{p}}{\delta v_{\bar{t}}^{\bar{p}}} \cdot v_{t}^{p} .
$$

The equivalent of the third assumption cannot be made to the cost functions of the links. It is very often the case that two distinct modes of transport (for example diesel and electrified trains) use the same infrastructure. That's why it is reasonable to assume that the interactions are limited to links which are parallel between two adjacent nodes $[8,14]$.

Now we shall introduce few additional notations. The link $a=(i, j, m)$ connects node $I$ with the node $j$ with the help of transport mode $m$. Let $M_{a}$ be the sub set of transport modes which we bear in mind in the computation of marginal costs for link a. Also let $A_{a} \doteq\left\{\tilde{a} \in A \quad \tilde{a}=\left(i, j, m^{\prime}\right)\right.$ or $\left.\tilde{a}=(j, i, m), m^{\prime} \in M_{a}\right\} \bigcup\{a\}$ be the set of links that must be considered in the computation of marginal costs for link a. Thus $s_{a}^{p}(v)=s_{a}^{p}\left(v_{\tilde{a}}^{p}\right), \tilde{a} \in A_{a}, p \in P[8,13,14]$.

If link $\tilde{a} \notin A_{a}$, then $\delta s_{a}^{p} / \delta \nu_{\tilde{a}}^{p}=0$. This permits the simplification of the equation (10) to the expression:

$$
c \frac{\bar{p}}{a}=s_{\bar{a}}^{\bar{p}}+\sum_{p \in P} \sum_{a \in H_{\bar{a}}} \frac{\delta s_{u}^{p}}{\delta v \frac{\bar{p}}{a}} \cdot v_{a}^{p}
$$

In this expression $H_{\bar{a}}=\left\{a \in A \mid \bar{a} \in A_{a}\right\}$. The set $H_{\bar{a}}$ can be get by scanning links parallel to $\bar{a}$ in both directions. This can take some time, particularly if $H_{\bar{a}}=\{\bar{a}\}$ for the majority of the links. That's why we make the last assumption that the cost of links depends only on the flows of links that share the same head and tail nodes and are in both orientations. This assumption is equivalent to $\mathrm{H}_{\mathrm{a}}=\mathrm{A}_{\mathrm{a}}$ for all $\mathrm{a} \in \mathrm{A}$ [8]. So after all that the marginal cost of the links can be written as: 


$$
c \frac{\bar{p}}{\bar{a}}=s_{\bar{a}}^{\bar{p}}+\sum_{p \in P} \sum_{u \in A_{\bar{u}}} \frac{\delta s_{a}^{p}}{\delta v_{\bar{a}}^{\bar{p}}} v_{a}^{p} .
$$

A few words about the several assumptions that we made. First of all these assumptions simplify the specification of the cost functions for the model and decrease the burden of computing the marginal costs. They also correspond to actual practice and data availability, since the significant part of cost functions used in transport industry are defined for particular facility (road segment, rail link, etc). The total transport cost is the sum of these link costs and, possibly, a fixed cost component (this cost component varies with the volume of goods, but does not depend on a distance traveled) needed to start the trip on a particular mode. $[8,13,14]$

\section{A Solution Algorithm}

The problem that we consider may be written in a compact form as:

$$
\operatorname{Min} F(v), v \in \Omega \text {. }
$$

It is assumed that $F(v)$ is a convex function, once differentiable on an open space that contains $\Omega$. This assumption is satisfied, for example, by average transfer link cost function of the type:

$$
s_{a}^{p}=\sum_{i} \alpha_{i}\left(\beta_{i}+v_{a}^{p}\right)^{z(i)}, \alpha_{i}>0, \beta_{i}>0,0 \leq z(i)<\infty .
$$

This example demonstrates that average cost function is not to be convex $(0<z(i)<1)$ for $\mathrm{F}(v)$ to be convex $[4-6,10,13]$.

To solve this problem the first order method (linear approximation method) is used. This method which is used for solving large scale network equilibrium problems has a sub-linear rate of convergence in the vicinity of the optimal solution. But it is proved to be robust in application which does not require a very precise solution [5]. Also, its straightforward adaptation for our model poses the problem of dimension. The vector $v$ is of dimension $n_{p}\left(n_{A}+n_{T}\right)$. The same is the dimension of the feasible direction of descent generated by the linear approximation method. This may render the application of the linear approximation method impracticable in many computa tions.

The structure of the model suggests natural decomposition by a product. $\Omega$ is the product $\Pi_{R} \Omega^{p}$, where $\Omega^{p}$ is a set of feasible flows of product $p$ on the sub networks $m(p) \in M(p)$. The descent direction of the linear approximation method is found minimizing the linear approximation of the objective function which in this case is $F^{p}\left(F=\sum_{p=1}^{n_{p}} F^{p}\right)$. This means that one would have to solve $n_{p}$ minor problems in one cycle of algorithm.

The algorithm can be characterized as Gauss-SeidelLinear Approximation Approach. For each product $\mathrm{p}$ a descent direction is computed for $v^{\mathrm{p}}$, which is of dimension $\left(\mathrm{n}_{\mathrm{A}}+\mathrm{n}_{\mathrm{T}}\right)$. When considering a particular product all the flow variabies pertaining to the other products remain at their previous value. For each product $\mathbf{p}$ a single iteration of the linear approximation algorithm is carried out. So the algorithm looks like that:

\section{Step 0. Initialization}

Determine $v$ (initial feasible solution)

Step 1. Major cycle

$v_{\text {old }}=v$; for each $\mathrm{p} \in \mathrm{P}$ carry out (1p).

(1p) Minor cycle

Compute $c^{\mathrm{P}}(v) \quad$ (marginal costs)

Find $\omega^{\mathrm{p}}$ (extreme point found by shortest path computation)

$D^{p}=\omega p-v^{p} \quad$ (descent direction)

Compute $\lambda_{p}$ (optimal step size)

$v^{p}=v p+\lambda_{p} d^{p} \quad$ (update flow for $p$ ).

\section{Step 2. Stopping criterion}

If $\left(v=v_{\text {old }}\right)$, return to Step 1

Since the algorithm is stated in a rather compact form, some comments on the details of some steps should be made.

The minor cycle ( $1 p)$ implements one iteration of the linear approximation method in the sub-space of flows related to the product $\mathrm{p}$. A descent direction is found minimizing the linear approximation of $F^{p}$ on $\Omega^{p}$, which is:

$$
\operatorname{Min} \nabla F^{p}(v) y, \text { where } y \in \Omega^{p} \text {. }
$$

Since $F(v)=s(v)^{T} v$, it follows that $\nabla F(v)=$ $s(v)+v s^{\prime}(v)$. If marginal costs are defined as $c^{p}(v) \doteq s^{p}(v)+s^{p^{\prime}}(v)^{T} v$, then sub problem of linear programming is:

$$
\text { Min } c^{p}(\mathrm{v})^{T} y \text {, where } y \in \Omega^{p} .
$$

As it is well known, the solution of this problem is obtained assigning the demand $\mathrm{g}^{\mathrm{m}(\mathrm{p})}$ on the shortest path corresponding the modes that belong to $m(p)$ computed with link costs $\mathrm{c}^{\mathrm{p}}(\mathrm{v})$.

The optimal step size $\lambda_{\mathrm{p}}$ is obtained by minimizing the function $\mathrm{F}$ in the direction $\left(0, \ldots, d^{p^{T}}, \ldots, 0\right)^{T}$. Exception is a case when

$$
c^{p}(v)^{T} d^{p}=0 \text {. Then } \lambda_{\mathrm{p}}=0 .
$$

The initial solution is obtained carrying out a major cycle with initial marginal costs corresponding to $v=0$ (which means $\mathrm{c}(\mathrm{o})$ ) and setting $\lambda_{\mathrm{p}}=1$ in each minor cycle (Ip).

The algorithm is stopped when the flows $v$ change after a major cycle was carried out. This means that the current solution is optimal $[5,8]$.

\section{The Algorithm for the Shortest Path Computation \\ In the multimodal network that we use for the formu-}


lation of multiproduct flow modeling the path on which more than one mode of transport operates can be constructed only if a mode to mode transfer is permitted at a node that belongs to the path $[4,5,12-14]$.

In order to introduce the algorithm for the shortest path computation we introduce the following notation. Let $c_{a}, a \in A$ ir $c_{t}, t \in T$ be the "lengths" of links and transfer links. The shortest path algorithm finds the shortest path from an origin point $\sigma \in \mathrm{O}$ to all destination points $d \in D$ in the sub networks defined by the sub set of transport modes $m(p) \in \mathbb{M}(p)$. In this algorithm the shortest path is retracted by means of pointing out the preceding link. In the algorithm the following notations are used: $b_{d}$ is the access link to the destination point $d, b_{a}$ is the predecessor of link $\alpha$; the lengths of the shortest paths are given by variables $u_{d}$ ir $u_{a}$, where $u_{a}$ is a the length of the shortest path from the origin point o to link $\alpha$, inclusive of $c_{a} \cdot \bar{A}$ represents the set of links which were labeled, but still do not have permanent labels. So then an algorithm for the shortest path (including transfer links) computation can be stated as:

Step 0. Initialization

Lengths:

$u_{d}=\infty, d \in D, u_{o}=0 ; u_{a}=\infty, a \in A$

Predecessors:

$b_{d}=-1, d \in D ; b_{0}=0 ; b_{u}=0, a \in A$

Links to be labeled:

$\bar{A}=\varnothing$

Dummy link:

$a=(i, \bar{j}, \bar{m})$ with $\bar{i}=0, j=d, \bar{m}=m(p)$;

$u_{\bar{a}}=0$

Go to step 4.

Step 1. Choice of the link to be labeled

If $\mathrm{A}=\varnothing$, then STOP.

Choose $\bar{a}=(\bar{i}, j, \bar{m})$ of A such that $u_{\bar{a}} \leq u_{a}$ for all $a \in \bar{A}$

Link $\bar{a}$ receives a permanent label: $\bar{A}=\bar{A}-\{\bar{a}\}$

If $\bar{j}$ is a transfer node, go to step 3 ; if $\bar{j}$ is a regular node, go to step 4; otherwise continue. node);

Step 2. Test of a "head" node $\bar{j}$ (for destination

If $u_{\bar{a}} \leq u_{j}$ then $u_{\bar{j}}=u_{\bar{a}} ; b_{\bar{j}}=\bar{a}$ Return to step 1.

Step 3. Test of successors with transfers

For each $a=(i, j, m)$, such that $i=j$ and $m \in m(p)$ do:

If there is a transfer $t=(\bar{a}, a)$ do:

if $u_{\bar{a}}+c_{t}+c_{a}<u_{a}$,

then $u_{a}=u_{\bar{a}}+c_{t}+c_{a} ; b_{a}=\bar{a} ; \bar{A}=\bar{A} \cup\{a\}$,

otherwise, if $m=\bar{m}$, do:

if $u_{\bar{a}}+c_{a}<u_{a}$,

then $u_{a}=u_{\bar{a}}+c_{a} ; b_{a}=\bar{a} ; \ddot{A}=\bar{A} \cup\{a\}$.

Return to step 1 .
Step 4. Scan of successors without transfers

For each $a=(i, j, m)$, such that $i=\bar{j}$ and $m=\bar{m}$ do:

if $u_{\bar{u}}+c_{a}<u_{a}$,

then $u_{a}=u_{\bar{a}}+c_{a} ; b=\bar{a} ; \ddot{A}=\bar{A} \cup\{a\}$.

Return to step 1

A few remarks regarding this shortest path algorithm should be made.

As a rule, each link corresponds to only one mode of transport.

The dummy link that has to do with the origin node o has its own set of transport modes $m(p)$, that have a permission to operate on this link.

The calculations are done in the way so that the first time when Step 4 is carried out all the links that go from o belong to the network. Thus, the first time that Step 4 is executed, the test $m=\bar{m}$ is $m \in \bar{m}=m(p)$.

In Step 3 links are scanned in an increasing order, so the transfer links of type $(\bar{a},$.$) are sorted in an increasing$ order as well.

The links that belong to the node $i$ are sorted by mode of transport in the "link table". Such an efficient test must preclude the scan of all the successors links in Step 4.

\section{Conclusions}

In the article a model for modelling freight flows that consist of multiple products transported by different means of transport in a multimodal network is formulated. This model can be used for the purpose of strategic freight flows planning. Besides, this model is enough generalpurpose, so it can be adapted for modeling national as well as regional freight flows. The original features of the model are:

- the way of network representation;

- the algorithm of mathematical solution;

- the adaptation of the shortest path search algorithm;

- the coupling of all that was mentioned above into the whole, what permits easy and effective analysis of flows of multiple products that move in a multimodal network.

Besides, the model is flexible enough to be used in various contexts. The most important thing is that model can be applied in a case of analysis of very large networks and freight flows.

\section{References}

1. Florian M. An introduction to network models used in transport planning. In: Transport Planning Models. Amsterdam: North-Holland. 1984, p 137-152.

2. Friesz T. L., Harker P. T. Freight network equilibrium: a review of the state of the art. In: Analytical studies in Transport Economics, chapter 7. Cambridge. 1985.

3. Friesz T. L., Gotfried J. A., Morlok E. K. A sequential 
shipper-carrier network model for predicting freight flows. Transportation Science, No 20, 1986, p 80-91.

4. Bronzini M. S. Evolution of multimodal freight transportation network model. Transport Research Forum, $21^{\text {st }}$ annua meeting, 1980.

5. Friesz T. L., Viton P. A., Tobin R. L. Economic and computational aspects of freight network equilibrium models: a synthesis. Regional Science, No 25(1), 1985.

6. Potts R. B., Oliver R. M. Flows in transportation networks. New York: Academic press, 1972.

7. Baublys A. Introduction to the theory of transport system (Transporto sistemos teorijos ivadas). Vilnius: Technika, 1997. 298 p (in Lithuanian).

8. Sharp G. P.A multi-commodity intermodal transportation model. In: Transport Research Forum, $20^{\text {th }}$ annual meeting. 1979.

9. Crainic T. G., Florian M., Leal J-E. A model for the strategic planing of national freight transportation by rail. Transportation Science, No 24, 1990, p 1-24.
10. Friesz T. L., Tobin R. L, Harker P. T. Predictive intercity freight network models. Transport Research, Part A, No 17, 1983, p 409-417.

11. Jones P. S., Sharp G. P. Multi-mode intercity freight transportation planning for underdeveloped regions. In: Transport Research Forum, 20 $0^{\text {th }}$ annual meeting, 1979.

12. Guelat J., Florian M., Crainic T. G. A multimode multiproduct network assignment model for strategic planning of freight flows. Transportation Science, No 24, 1990, p 25-39.

13. Harker P. T., Friesz T. L. Prediction of intercity freight flows I: Theory. Transport Research Part B, No 20, 1986, p 139153.

14. Harker P. T. Friesz T. L. Prediction of intercity freight flows II: Mathematical formulations. Transport Research Part B, No 20, 1986, p 155-174. 